\title{
DYNAMICS OF SOLITONS AND QUASISOLITONS OF CUBIC THIRD-ORDER NONLINEAR SCHRÖDINGER EQUATION
}

\author{
V.I. Karpman ${ }^{1}$, J.J.Rasmussen ${ }^{2}$ and A.G. Shagalov ${ }^{3}$ \\ ${ }^{1}$ Racah Institute of Physics, Hebrew University of Jerusalem, Jerusalem 91904, Israel \\ Dept. of Optics and Fluid Dynamics, Risoe National Laboratory, Box 49, DK-4000 Roskilde, Denmark, \\ ${ }^{3}$ Institute of Metal Physics, Ekaterinburg 620219, Russian Federation.
}

\begin{abstract}
The dynamics of soliton and quasisoliton solutions of cubic third order nonlinear Schrödinger equation is studied. The regular solitons exist due to a balance between the nonlinear terms and (linear) third order dispersion; they are not important at small $\alpha_{3}\left(\alpha_{3}\right.$ is the coefficient in the third derivative term) and vanish at $\alpha_{3} \rightarrow 0$. The most essential, at small $\alpha_{3}$, is a quasisoliton emitting resonant radiation (resonantly radiating soliton). Its relationship with the other (steady) quasisoliton, called embedded soliton, is studied analytically and in numerical experiments. It is demonstrated that the resonantly radiating solitons emerge in the course of nonlinear evolution, which shows their physical significance.
\end{abstract}

PACS numbers: 42.65.Tg, 52.35.Mw

\section{INTRODUCTION}

Equations describing soliton processes are usually obtained by certain approximating procedures affecting nonlinearity and dispersion. Now, in extensive studies of ultrafast processes, the classical approximations often appear to be insufficient and higher order effects become of importance. A typical example is high speed systems like nonlinear transmission lines in femtosecond regime for soliton communications, etc. For such systems soliton solutions in classical sense do not generally exist. Only for very specific choices of parameters one can find localized solutions. Instead of regular solitons there may appear nonlocal steady or (and) unsteady soliton like structures (which may be called quasisolitons). These may, however, have significant importance for the nonlinear dynamics.

In this paper we consider dynamics described by the extended third-order cubic nonlinear Schrödinger (NLS) equation

$$
\begin{gathered}
i \partial_{T} \Psi+\frac{1}{2} \partial_{X}^{2} \Psi+|\Psi|^{2} \Psi+i \alpha_{1}|\Psi|^{2} \partial_{X} \Psi+i \alpha_{2} \Psi \partial_{X}|\Psi|^{2} \\
+i \alpha_{3} \partial_{X}^{3} \Psi=0
\end{gathered}
$$

which plays an important role in many nonlinear problems, in partiqular, in the nonlinear fiber optics $[1,2]$. The third derivative term describes higher order correction to the linear dispersion and the cubic terms with the first derivative are dispersive nonlinear corrections. Here, we assume that all $\alpha_{n}\left(1 \leq \alpha_{n} \leq 3\right)$ are real parameters.

Eq.(1) has regular soliton solutions [3-5], vanishing at $|X| \rightarrow 0$. In particular cases, Eq.(1) may have exact $N$ soliton solutions [4] or even be integrable [5]. An important feature of the solutions describing regular solitons is that they are degenerating at $\alpha_{3} \rightarrow 0$ (see below), which substantially reduces their physical importance. Their numerical investigation at $\alpha_{1,2} \sim 1$ where done e.g. in Ref.[6] (see also references therein)
There also exist some solutions to Eq.(1), describing quasisolitons. One of them is a steady solution looking like a soliton type pulse embedded into a small amplitude plane wave (a soliton on plane wave pedestal). We call it embedded soliton (ES), using the word proposed in a different context in Ref.[7]. Apart from that, Eq. (1) has other type of quasisoliton solutions which describe soliton-like pulses permanently emitting resonantly generated radiation (see, e.g., Refs. [8-12] for the particular case at $\alpha_{1}=\alpha_{2}=0$ and $[13,14]$ for the full Eq.(1)). We will call them resonantly radiating solitons (RRS); they are unsteady because of losses caused by the radiation. The lifetime of radiating soliton is sufficiently large if $\alpha_{3}$ is small enough and, naturally, one can speak about the soliton only in this quasisteady case. (However, at long times, the losses caused by the radiation become essential for applications [2].) At $\alpha_{3} \rightarrow 0$, the RRS turns into the regular soliton of Eq.(1) without the third derivative.

There is an interesting and important connection between the two types of quasisolitons, ES and RRS, and we demonstrate it in numerical simulations. We will also study the role of solitons and quasisolitons in the nonlinear processes described by Eq.(1). In partiqular, the regular solitons and RRS compete between themselves in nonlinear processes. As far as the regular solitons disappear at $\alpha_{3} \rightarrow 0$ and the RRS have short lifetimes at large $\alpha_{3}$, it is clear that at large $\alpha_{3}$ the regular solitons are more important while the RRS may play a decisive role at sufficiently small $\alpha_{3}$. The most interesting case is, of course, small $\alpha_{3}$, because the third derivative term emerges as the result of an expansion. (The effect of next, 4th derivative, term can be seen in Refs.[13,14]; at certain relalionship between the coefficients before third and fourth derivatives there can be no radiation at all which, naturally, may happen also when other high-order terms are taken into account.)

The paper is organized as follows. In Sec.I we describe important properties of Eq.(1), which are used below. In particular, we discuss regular soliton solu- 
tions [3], Galilean transformations and conservation laws $[13,14]$. The embedded solitons are studied in Sec.3. As we have already mentioned, the ES consists of a steady soliton-like pulse on the plane wave background. This structure is rater common in different nonlinear highly dispersive systems [9-20]. At $\alpha_{3} \rightarrow 0$, the plane wave disappears and the pulse turns into a regular soliton of Eq. (1) at $\alpha_{3}=0$. The plane wave amplitude, increasing with $\alpha_{3}$, may become unstable at sufficiently large $\alpha_{3}$ due to the modulational instability. Considering small $\alpha_{3}$, we show that the pulse part of ES is rather close to the pulse in RRS and the wavenumber of the plane wave coincides with the wavenumber of resonantly emitted radiation (by the RRS) . Next, we introduce the cut off operation, cutting off both wave wings from the ES (Sec.IV). Then we see that the remained soliton-like pulse is transformed into the RRS, emitting radiation only in one direction, according to the direction of the group velocity of resonant radiation. The resonant radiation disappears at $\alpha_{1}=6 \alpha_{3}$. In this case, considered in Sec.V, we show that Eq.(1) can be transformed, by means of the Galilean transformation, to the complex modified Korteweg-de Vries (MKdV) equation . At particular initial conditions, it is reduced to the real $\mathrm{MKdV}$ equation which is integrable and therefore has $N$ - soliton solutions [22]. This does not mean the complete integrability of Eq.(1) at $\alpha_{1}=6 \alpha_{3}$, because the reduction to the real MKdV equation is possible only for particular initial conditions. However, the Painlevé analysis [21] of the above mentioned complex MKdV equation shows that at $6 \alpha_{3}=\alpha_{1}=2 \alpha_{2}$ it possesses the Painlevé property. This is just the case when Eq. (1) is integrable [5]. If $\alpha_{2}=0$, we arrive at the case considered by Hirota [4] who has shown the existence of $N$ - soliton solutions in this case. Our approach, however, is more general and (in fact, as in Ref. [23] ) we can see that the $N$ -soliton solutions are possible even for nonvanishing $\alpha_{2}$ (at $\alpha_{2} \neq-3 \alpha_{3}$ ). Then we present a numerical study of the initial value problem in the Hirota case: $\alpha_{1}=6 \alpha_{3}$, $\alpha_{2}=0$ taking as initial conditions complex pulses and one can see how the robust Hirota solitons are emerging from these pulses. In Sec.VI, we describe numerical experiments at $\alpha_{1} \neq 6 \alpha_{3}$. Solving the initial value problem, we show that, at sufficiently small $\alpha_{3}$, the initial disturbances decay into the resonantly radiating solitons (RRS). On the other hand, at small $\alpha_{3}$, the Potasek- Tabor solitons [3] were not detected. This indicates that the RRS are important physical objects playing a significant role in nonlinear dynamics. In Sec. VII, a summary of obtained results is given. In Appendix A, the modulational instability of a plane wave, described by Eq.(1), is considered; it is helpful in the study of the stability of embedded solitons. In Appendix B we investigate analytically, by means of conservation laws, the evolution of RRS caused by the radiation. This analysis is in agreement with numerical results described in Secs.IV and VI.

\section{IMPORTANT PROPERTIES OF EQ. (1)}

\section{a. Exact soliton solutions}

First, we discuss the exact soliton solutions of Eq.(1). They can be written in the form

$$
\begin{gathered}
\Psi_{s}=a \operatorname{sech}\left[b\left(X-V_{s} T\right)\right] e^{i k X-i \omega T}, \\
a^{2}=\frac{6 \alpha_{3}}{\alpha_{1}+2 \alpha_{2}} b^{2}\left(\alpha_{1} \neq-2 \alpha_{2}\right), \\
V_{s}=\kappa-3 \alpha_{3} \kappa^{2}+\alpha_{3} b^{2}, \\
\omega=\frac{1}{2} \kappa^{2}-\alpha_{3} \kappa^{3}-\frac{1-6 \alpha_{3} \kappa}{2} b^{2}, \\
\kappa=\frac{\alpha_{1}+2 \alpha_{2}-6 \alpha_{3}}{12 \alpha_{2} \alpha_{3}} .
\end{gathered}
$$

In fact, these are the soliton solutions found by Potasek and Tabor [3] (with corrected misprints). We will call Eqs. (2a-e) Potasek-Tabor (PT) soliton solutions. At $\alpha_{3}=0$, PT solitons do not exist; this signifies that their existence is a result of a ballance between the nonlinear terms and the linear third order dispersive term. If $\alpha_{2} \neq 0$, from Eq. (2e) it follows that $\kappa$ is a fixed number, uniquely determined by the coefficients of Eq.(1). However, the solitons (2) exist even at $\alpha_{2}=0$ provided that

$$
\alpha_{1}=6 \alpha_{3}
$$

In this case $\kappa$ can be arbitrary, because Eq. (2e) follows from the equation $12 \alpha_{2} \alpha_{3} \kappa=\alpha_{1}-6 \alpha_{3}+2 \alpha_{2}$.

In case (3) and $\alpha_{2}=0$, Eq. (1) is the so called Hirota equation that can be transformed to the complex modified Korteweg-de Vries equation which has $N$-soliton solutions [4]. For the Hirota soliton, from Eq.(2b) it follows

$$
b=a \text {. }
$$

In the other particular case, $6 \alpha_{3}=\alpha_{1}=2 \alpha_{2}$, Eq. (1) is integrable [5]. (See also Sec.V.) Some results for solitons (2) with $\alpha_{2} \neq 0$ were reported in Ref.[6] (see also references therein). In particular, it was shown numerically that they emerge in a solution of initiall value problem. However, this result was obtained at $\alpha_{1}=\alpha_{3}=1$; at small $\alpha_{3}$, as it will be shown below, they cannot compete with RRS.

Apart from the solution (2a-e), describing "bright" solitons, which was called in Ref. [3] sech-family, Potasek and Tabor have also found tanh-family which will not be discussed here. (Some generalizations, with both sech and tanh terms, are derived in Ref. [27] by means of rather tedious algebra; the results of this paper are mostly contained in Ref.[3] and the generalizations [27], 
which are valid for very specific choices of the parameters, can be obtained by the much simpler approach of Potasek and Tabor.) The results of Ref.[3] are repeated also in some other papers (e.g.[23]).

\section{b. Galilean transformation}

In the following we shall frequently use the Galilean transformation describing the transition to the reference frame moving with the velocity $V$. For Eq. (1) it reads

$$
\Psi(X, T)=\psi(X-V T, T) \exp [i(K X-\Omega T)],
$$

where $K$ and $\Omega$ are defined by equations

$$
\begin{gathered}
V=K-3 \alpha_{3} K^{2}, \\
\Omega=\frac{1}{2} K^{2}-\alpha_{3} K^{3} .
\end{gathered}
$$

The function $\psi(x, t)$ with

$$
x=X-V T, t=T
$$

satisfies the equation $[13,14]$

$$
\begin{gathered}
i \partial_{t} \psi+\frac{1}{2} a_{2} \partial_{x}^{2} \psi+q|\psi|^{2} \psi+i \alpha_{1}|\psi|^{2} \partial_{x} \Psi+i \alpha_{2} \psi \partial_{x}|\psi|^{2} \\
+i \alpha_{3} \partial_{x}^{3} \psi=0
\end{gathered}
$$

with

$$
\begin{gathered}
a_{2}=1-6 \alpha_{3} K, \\
q=1-\alpha_{1} K .
\end{gathered}
$$

\section{c. Conservation laws}

One can check by means of straightforward calculations that Eq. (1) has the following integral of motion

$$
N=\int_{-\infty}^{\infty}|\Psi(X, T)|^{2} d X
$$

At

$$
\alpha_{2}=0
$$

we have two other conserving integrals $[24,13,14]$

$$
\begin{gathered}
P=\frac{1}{2} i \int_{-\infty}^{\infty}\left(\Psi \partial_{X} \Psi^{*}-\Psi^{*} \partial_{X} \Psi\right) d X \\
H=\int_{-\infty}^{\infty}\left\{\frac{1}{2}\left|\partial_{X} \Psi\right|^{2}-\frac{1}{2}|\Psi|^{4}-\frac{1}{4} i \alpha_{1}|\Psi|^{2}\left(\Psi^{*} \partial_{X} \Psi-\Psi \partial_{X} \Psi^{*}\right)\right.
\end{gathered}
$$

$$
\left.-\frac{1}{2} i \alpha_{3}\left(\Psi^{*} \partial_{X}^{3} \Psi-\Psi \partial_{X}^{3} \Psi^{*}\right)\right\} d X
$$

\section{EMBEDDED SOLITONS}

We start with the solutions to Eq. (1) of the form

$$
\Psi(X, T)=\chi(x) e^{i \Lambda t},
$$

where $x$ and $t$ are defined in (8). Substituting (16) into Eq.(1), we arrive at the ordinary differential equation

$$
-i V \partial_{x} \chi+\frac{1}{2} \partial_{x}^{2} \chi+|\chi|^{2} \chi+i \alpha_{1}|\chi|^{2} \partial_{x} \chi+i \alpha_{2} \chi \partial_{x}|\chi|^{2}
$$

$$
+i \alpha_{3} \partial_{x}^{3} \chi=\Lambda \chi .
$$

Imposing periodic boundary conditions at the ends of a sufficiently broad interval and considering $V$ and $\chi(0)$ as given parameters and $\Lambda$ as the eigenvalue, we arrive at a nonlinear eigenvalue problem which can be numerically solved by a kind of shooting method. An example of such solution at $\alpha_{2}=0$ and

$$
\alpha_{1}=-0.3, \quad \alpha_{3}=-0.1, \quad \chi(0)=1.5, \quad V=0
$$

is shown in Fig.1(a,b) . It is a nonlocal steady pulse with $|\Psi(x)|_{\max }=\left|\Psi\left(x_{0}\right)\right|$ and small symmetric "wings". At large $\left|x-x_{0}\right|$ we can linearize Eq. (17); then we see that

$$
\chi(x) \approx \operatorname{const} e^{i \kappa x} \quad\left(\left|x-x_{0}\right| \gg 1\right),
$$

where $\kappa$ is a real root of cubic algebraic equation

$$
\Lambda-\kappa V+(1 / 2) \kappa^{2}-\alpha_{3} \kappa^{3}=0 .
$$

Finding $\Lambda$ from the numerical solution of the eigenvalue poblem and $\kappa$ from (20), we have for the case (18)

$$
\Lambda=0.979, \quad \kappa=-5.34 \text {. }
$$

On the other hand we can determine $\kappa$ directly from $\chi(x)$ which is found numerically from Eq. (17), together with $\Lambda$. From Fig. 1b we see that the numerically found $\chi(x)$ has indeed asymptotic behavior (19) with $\kappa \approx-5.3$ which is very close to the root of Eq. (20), written in (21). This agreement is an evidence of the correctness of the numerical solution of eigenvalue problem by the shooting method. In a similar way, the solution of eigenvalue problem (17) and Eq. (20) for $\alpha_{2}=0$ and

$$
\alpha_{1}=-0.8, \quad \alpha_{3}=-0.1, \quad \chi(0)=1.5, \quad V=-0.225
$$

gives

$$
\Lambda=1.209, \quad \kappa=-5.03 .
$$


FIG. 1. Numerical solution of Eq. (17) at parameters (18). (a) $\chi$ versus $x$; solid line: $|\chi(x)|=|\Psi(x)|$; dashed line: $\operatorname{Re} \chi(x)$. (b) $\arg \chi$ versus $x$; at sufficiently large $\left|x-x_{0}\right|$, $\arg \chi(x)$ is a linear function: $\arg \chi \approx \kappa\left|x-x_{0}\right|$, which permits to measure $\kappa(\kappa \approx-5.2)$.

The asymptotic behavior of numerically found $\chi(x)$ gives the same $\kappa \approx-5.03$.

For the other case with $\alpha_{2}=0$ and

$$
\alpha_{1}=-1.1, \quad \alpha_{3}=-0.1, \quad \chi(0)=1.5, \quad V=0.35
$$

we have

$$
\Lambda=1.937, \quad \kappa=-6.12 \text {. }
$$

while from the numerically found $\chi(x)$ we obtain $\kappa=$ -6.1 .

In fact, similar quasisoliton solutions, looking like embedded solitons, were obtained, by different approaches, for other highly dispersive systems as well [15$17,9,11,12,17-20]$.

Now consider the embedded solitons, starting from Galilean transformation (5). Writing it in the form

$$
\Psi(X, T)=\psi(x, t) e^{i K x} \exp [i(K V-\Omega) t]
$$

and assuming that

$$
\psi(x, t)=\tilde{\psi}(x) \exp \left(i \frac{1}{2} \lambda^{2} t\right),
$$

where $\lambda$ is a constant parameter, we compare (24) with (16). Then we have

$$
\begin{gathered}
\tilde{\psi}(x)=\chi(x) \exp (-i K x), \\
\lambda^{2}=2(\Lambda+\Omega-K V) .
\end{gathered}
$$

From (25) and (9) we arrive at the following equation for $\psi \tilde{(x)}$

$$
\begin{gathered}
-\frac{1}{2} \lambda^{2} \tilde{\psi}+\frac{1}{2} a_{2} \partial_{x}^{2} \tilde{\psi}+q|\tilde{\psi}|^{2} \tilde{\psi}+i \alpha_{1}|\tilde{\psi}|^{2} \partial_{x} \tilde{\psi}+i \alpha_{2} \tilde{\psi} \partial_{x}|\tilde{\psi}|^{2} \\
+i \alpha_{3} \partial_{x}^{3} \tilde{\psi}=0
\end{gathered}
$$

Substituting here (26), (27) and taking into account (6), (7) and (10), (11) we have Eq.(17) as one should expect.
From Eq. (26) it is seen that $|\tilde{\psi}|^{2} \ll 1$ at $|x| \gg 1$. Linearizing Eq. (28) we obtain

$$
\tilde{\psi} \sim e^{i k x} \quad(|x| \gg 1),
$$

where $k$ is a root of the equation

$$
2 \alpha_{3} k^{3}-a_{2} k^{2}-\lambda^{2}=0 .
$$

Substituting (19) and (29) into Eq. (26), we see that

$$
\kappa=k+K \text {. }
$$

Then, using (6), (7) and (10), (27) we can easily prove that Eqs. (30) and (20) are equivalent, as it should be.

At small $\alpha_{3}$, the solution of Eq.(28) can be written as

$$
\tilde{\psi}(x)=\left[u_{s}(x)+f(x)\right] \exp \left[i \phi_{s}(x)\right] .
$$

with small $f(x)$. Here $u_{s}(x)$ and $\phi_{s}(x)$ are defined by the requirement that

$$
F(x)=u_{s}(x) \exp \left[i \phi_{s}(x)\right]
$$

is a soliton solution of Eq. (28) without the last term i.e.

$-\frac{1}{2} \lambda^{2} F+\frac{1}{2} a_{2} \partial_{x}^{2} F+q|F|^{2} F+i \alpha_{1}|F|^{2} \partial_{x} F+i \alpha_{2} F \partial_{x}|F|^{2}=0$

and $F(x) \rightarrow 0$ at $x= \pm \infty$. Solving Eq. (34), we have

$$
\begin{gathered}
u_{s}(x)=\lambda \sqrt{\frac{2 p}{q}}\left[\cosh \left(\frac{2 \lambda}{\sqrt{a_{2}}} x\right)+p\right]^{-1 / 2} \\
\phi_{s}(x)=-\frac{\alpha_{1}+2 \alpha_{2}}{2 A} \arctan \left[\sqrt{\frac{1-p}{1+p}} \tanh \left(\frac{\lambda x}{\sqrt{a_{2}}}\right)\right] \\
p=\frac{\sqrt{a_{2}} q}{\sqrt{4 A^{2} \lambda^{2}+a_{2} q^{2}}}
\end{gathered}
$$

$$
A^{2}=\frac{4 \alpha_{1}\left(\alpha_{1}+2 \alpha_{2}\right)-\left(\alpha_{1}+2 \alpha_{2}\right)^{2}}{12} .
$$

Eqs. (35)-(38) were obtained in Ref. [14] for a pulse part of RRS. From (35) it follows that the soliton amplitude is

$$
u_{0}=\lambda \sqrt{\frac{2 p}{(1+p) q}}
$$

and its width is given by

$$
\delta=\sqrt{a_{2}} / \lambda .
$$

Thus one must require $a_{2}>0$ and from Eq. (10) it follows that at $\alpha_{3} K>0$,

$$
|K|<\frac{1}{6}\left|\alpha_{3}\right|,
$$


which is a restriction on the soliton velocity (6).

A small term $f(x)$ in Eq.(32) expresses the effect of the third order dispersion. At $\left.|x| \sim \sqrt{(} a_{2}\right) / \lambda$, or less, it describes a modification of the pulse, arising due to the last term in Eq. (28), while at large $x$

$$
f(x) \approx \tilde{\psi}(x) \sim e^{i k x}
$$

where $k$ is a root of Eq. (30). Note, that this equation was derived in Ref. [14] for the wavenumber of the resonantly generated radiation by the RRS in the reference frame where the RRS is at rest. From all that we conclude that (32) coincides with the asymptotic expression for the RRS and its radiation at large $t$.

Let us now compare the embedded solitons, obtained above numerically, with the solution (32) at condition (13) . From Eq.(38) we have

$$
A=\frac{1}{2}\left|\alpha_{1}\right| .
$$

Eqs. (6) and (10) give

$$
\begin{gathered}
K=\frac{1-\sqrt{1-12 \alpha_{3} V}}{6 \alpha_{3}}, \\
a_{2}=\sqrt{1-12 \alpha_{3} V} .
\end{gathered}
$$

Using (39) and (37), we obtain

$$
u_{0}^{2}=\frac{\sqrt{a_{2}}}{2 A^{2}}\left(\sqrt{4 A^{2} \lambda^{2}+q^{2} a_{2}}-q \sqrt{a_{2}}\right),
$$

where $q$ is defined in (11). From (27) and (6),(7) we find

$$
\lambda^{2}=2\left(\Lambda-\frac{1}{2} K^{2}+2 \alpha_{3} K^{3}\right) .
$$

Then we have for the case (18), (21), which is shown in Fig. $1: u_{0} \approx 1.37$. For the case $(22) u_{0} \approx 1.41$, and for (23) $u_{0} \approx 1.42$. So, in all three cases we approximately have $u_{0} \approx 1.4$ which is rather close to $\chi(0)=1.5$, assumed in the nonlinear eigenvalue problem for all three cases. This supports the conclusion that expressions (35) and (36) approximately describe the pulse in the embedded soliton (at small $\alpha_{3}$ ).

Now consider $f(x)$. At small $\alpha_{3}$, the roots of Eq. (30) have simple analytical expressions [14]. Neglecting the first term in (30), we have two smallest roots

$$
k \approx \pm i \frac{\lambda}{\sqrt{a_{2}}} .
$$

Substituting this into (42), we have

$$
\tilde{\psi} \approx f(x) \sim \exp \left(\mp \lambda / \sqrt{a_{2}} x\right) .
$$

This is in agreement with the asymptotic behavior of expression (33) at large $x$. The third root can be approximately obtained if one neglects the last term in (30). This gives

$$
k \approx a_{2} / 2 \alpha_{3},
$$

which approximately determines the wavenumber of the plane wave in the wings. Expressions (50) and (31) approximately give the roots of Eq. (20) in analytical form. From (50) it follows

$$
\operatorname{sgn} k=\operatorname{sgn} \alpha_{3} .
$$

And, finally, from the results of Ref. [14] it follows that at small $\alpha_{3}$

$f(x) \approx B\left(\frac{\sqrt{a_{2}}|k|}{A}\right)^{\frac{1}{2}} \exp \left[-\frac{\pi \sqrt{a_{2}}|k|}{4 \lambda}\left(1+\frac{2}{\pi} \arcsin p\right)\right] e^{i k x}$,

where $B$ is a complex constant with $|B| \sim 1-10$. This expression is valid at

$$
\frac{\sqrt{a_{2}}|k|}{\lambda} \gg 1
$$

i.e. when the wavenumber $k$ is much larger than the inverse width of the soliton (33). In expression (52), it is also assumed that $2 A \lambda \gg \lambda / \sqrt{a_{2}}\left|k_{1,2}\right|$ (this does not exclude $A \lambda \ll 1)$. If

$$
2 A \lambda \sim \lambda / \sqrt{A_{2}}\left|k_{1,2}\right| \quad\left(\text { or } 2 A \lambda<\lambda / \sqrt{a_{2}}\left|k_{1,2}\right|\right),
$$

which may be satisfied only at $p \approx 1$ and $q \approx 1$, we have

$$
f(x)=B \sqrt{a_{2}}|k| \exp \left(-\frac{\pi \sqrt{a_{2}}|k|}{2 \lambda}\right) e^{i k x} .
$$

It is easy to check that we can arrive at Eq. (55) by substituting in Eq. (52) the first of conditions (54) and $p=1$.

From the all foregoing, one can see a connection between the embedded and resonantly radiating solitons. In the next section we present numerical experiments disclosing this connection from another side.

\section{THE CUT OFF OPERATION}

Let us define the cut off operation transforming the embedded soliton into an isolated pulse. Turning to the function $\chi(x)$ in (16), we write

$$
\chi_{c u t}(x)=\chi(x) r(x),
$$

where $r(x)$ is a cutting factor which we take in the form

$r(x)=\frac{1}{2}\left[\tanh \left(\frac{x-x_{0}+\Delta x}{\gamma}\right)-\tanh \left(\frac{x-x_{0}-\Delta x}{\gamma}\right)\right]$.

Here $x_{0}$ is the center of the pulse and $\Delta x>0$ is the width of the cutted function $\chi_{c u t}(x)$. According to (57), $r(x)$ vanishes at $\left|x-x_{0}\right| \rightarrow \infty$ and the positive parameter $\gamma$ characterizes the "sharpness" of vanishing. Assuming that $\gamma$ is small enough and $\Delta x$ is such that the factor $r(x)$ cuts off only the wings without essential disturbing the pulse in $\chi(x)$ ( $\gamma$ and $\Delta x$ can be properly chosen in 
numerical tests) we then take the pulse $\chi_{\text {cut }}(x)$ as the initial condition to Eq.(1) (see Fig.2a)

$$
\Psi(X, 0)=\chi_{c u t}(X) .
$$

In Fig.2b one can see that the cutted pulse emits radiation at $t>0$. However, on the left hand side of the pulse the radiation spreads out with time; this shows that on the left hand side there is a transient radiation, emitted at small $t$ due to initial condition (58). On the contrary, on the right hand side we see a wave train with approximately constant amplitude, with the front propagating to the right; so, the length of the wavetrain increases with time. Therefore the cutted pulse permanently emits radiation to the right. The spectrum, shown in Fig.1c, has a peak at $\kappa$ approximately equal to the root of Eq. (20) with account of finite velocity of the pulse at $t=138$ (it is still rather close to $\kappa$ from Eq. (21), i.e. to the wavenumber of the wing waves in embedded soliton).

Analyzing the time behavior of $\arg \Psi$, we find that $d(\arg \Psi) / d t=\Lambda(t)$ is a slow function of $t$, with $\Lambda(0) \approx$ 0.98 and $\Lambda(130) \approx 0.85$. Note that this $\Lambda(0)$ coincides, with a good accuracy, with (21). Similar results were obtained for cutted pulses in cases (22) and (23).

We conclude that the cutted pulses are radiating solitons; and the radiation is permanently emitted only in one direction. As far as $\kappa$ is connected with $k$ from Eq. (31), which is the real root of Eq. (30), obtained from the resonant condition, we conclude that the cut off operation transforms ES ino RRS. The front of radiated wavetrain propagates with the group velocity $U(k)$ given by $[14]$

$$
U(k)=a_{2} k-3 \alpha_{3} k^{2} \approx-a_{2} / 4 \alpha_{3} .
$$

FIG. 2. Numerical solution of Eq. (1) at initial condition (58) and $\alpha_{1}=-0.3, \alpha_{2}=0, \alpha_{3}=-0.1$. (a) The initial cutted pulse. Full line: $|\Psi|$ versus $x$ at $T=0$; dotted line depicts the corresponding embedded soliton. (b) The cutted pulse at $T=138$; full line: $|\Psi|$ versus $x$; dashed line: $\operatorname{Re} \Psi$ versus $x$. (c) $\log _{10}\left|\Psi_{k}\right|$ versus $\kappa\left(\Psi_{k}\right.$ is the Fourier transform of $\Psi(x)$; one can see a peak near the root of resonance equation (20).

From this it follows that

$$
\operatorname{sgn} U(k)=-\operatorname{sgn} k=-\operatorname{sgn} \alpha_{3} .
$$

Therefore at $\alpha_{3}<0$ it should be $k<0$ and $U(k)>0$. This means that the soliton in Fig. 2a should permanently emit radiation to the right while the peak in the spectrum of the wavetrain should be at negative $k$. This is in agreement with the results presented in Figs. $2 \mathrm{~b}$ and 2c.

To describe analytically the whole system after the cut off, one can use at small $\alpha_{3}$ the equation [14]

$$
\tilde{\psi}(x, t)=\left[u_{s}(x)+\eta(x, t)\right] \exp \left[i \phi_{s}(x)\right],
$$

where $u_{s}(x)$ and $\phi_{s}(x)$ are given (in adiabatic approximation) by Eqs. (35) and (36) and $\eta(x, y)$, at large $x$ and $t$, has the following asymptotic expression

$$
\eta(x, t) \approx f(x) \Theta(U x) \Theta(|U| t-|x|) .
$$

Here $f(x)$ is given by Eq. (52) and $\Theta(Y)$ is the Heaviside function

$$
\Theta(Y)=1(Y>0), \Theta(Y)=0(Y<0), .
$$

Eq. (62) expresses that the soliton radiates in the direction of group velocity $U$ and the radiation front propagates with the velocity $|U|$. The mentioned above adiabatic approximation means that at sufficiently small $\alpha_{3}$, the radiation is so small that the soliton parameters in $u_{s}(x)$ and $\psi_{s}(x)$ (as well as the wavenumber $k$ ) can be considered as constant. However, the soliton losses may be essential at large times.

FIG. 3. Temporal behavior of the radiating soliton at $\alpha_{1}=-0.3, \alpha_{2}=0, \alpha_{3}=-0.1$. (a) $|\Psi|_{\max }$ versus $T=t$; (b) the soliton position versus $t$.

The variation of soliton parameters, caused by the radiation, can be estimated by means of the integrals of motion (Appendix B). A vast information about the soliton evolution, caused by the radiation, follows from the numerical solution of Eq.(1). For the parameters used in Figs. 2, the results following from this solution are presented in Fig.3. One finds that the mean value of $|\Psi|_{\max }=u_{0}(t)$, shown in Fig.3a, decreases logarithmically, similar to what was found for the case $\alpha_{1}=\alpha_{2}=0[25,26]$. Analyzing the soliton position (Fig.3b) one can find that the soliton velocity $V(t)$ increases from $V(0)=0$ to $V(138) \approx 0.29$, i.e. the soliton is accelerating and the function $V(t)$ increases (also logarithmically). Now, using $\Lambda(0) \approx 0.98, \Lambda(138) \approx 0.85$ and Eqs. (47),(44), we have $\lambda(0) \approx 1.4, \lambda(138) \approx 1.28$. Therefore, the soliton width $\Delta=\sqrt{a_{2}} / \lambda$ increases from $\Delta(0) \approx 0.71$ to $\Delta(138) \approx 0.99$, i.e. the radiating soliton is widening with time. All that can be considered as a numerical confirmation of analytical results obtained in Appenix B from the conservation laws, in particular that

$$
\operatorname{sgn} \frac{d V}{d t}=-\operatorname{sgn} k=\operatorname{sgn} U
$$


which means that the soliton is accelerating in the direction of the group velocity of resonant radiation.

V. SPECIAL CASE $\alpha_{1}=6 \alpha_{3}$. HIROTA SOLITONS

The coefficient $q$ in Eq. (9) disappears at

$$
K=1 / \alpha_{1} .
$$

Substituting this in Eq. (10), we have $a_{2}=1-6 \alpha_{3} / \alpha_{1}$. Therefore, at condition (3) the coefficient $a_{2}$ also vanishes and Eq. (9) takes the form

$$
\partial_{t} \psi+6 \alpha_{3}|\psi|^{2} \partial_{x} \psi+\alpha_{2} \psi \partial_{x}|\psi|^{2}+\alpha_{3} \partial_{x}^{3} \psi=0 \text {. }
$$

which is related to Eq.(1) by a Galilean transformation (5),(8) with

$$
V=\frac{1}{12 \alpha_{3}}, \quad K=\frac{1}{6 \alpha_{3}}, \quad \Omega=\frac{1}{108 \alpha_{3}^{2}} .
$$

Consider a particular solution of Eq.(66)

$$
\psi(x, t)=e^{i \theta} \zeta(x, t)
$$

where $\theta=$ const and $\theta, \zeta(x, t)$ are real. Then Eq. (66) is reduced to the modified Korteweg-de Vries (MKdV) equation

$$
\partial_{t} \zeta+2\left(3 \alpha_{3}+\alpha_{2}\right) \zeta^{2} \partial_{x} \zeta+\alpha_{3} \partial_{x}^{3} \zeta=0
$$

It is completely integrable and, in particular, has exact $N$ - soliton solutions [22] if $\alpha_{2} \neq-3 \alpha_{3}=-(1 / 2) \alpha_{1}$ [cf. with Eq. (2b)]. As far as Eq. (66) is reduced to Eq. (69) only for particular initial conditions, it may not be , generally, integrable; however, one can assert that it also possess $N$ - soliton solutions at any $\alpha_{2} \neq-(1 / 2) \alpha_{1}$, which is a generalization of the Hirota solution at $\alpha_{2}=0$. Below, we call Eq. (66) complex MKdV equation. The Painlevé test [21], applied to Eq. (66), shows that it has the Painlevé property if $\alpha_{2}=3 \alpha_{3}$, i.e.

$$
6 \alpha_{3}=\alpha_{1}=2 \alpha_{2} \text {. }
$$

This is just the integrability condition for Eq.(1) found by Sasa and Satsuma [5]. These conclusions are in agreement with those following from the Painlevé test of Eq.(1) $[28,23]$.

If, in addition to condition (3), $\alpha_{2}=0$ (Hirota case [4]), we arrive at the complex MKdV equation

$$
\partial_{t} \psi+6 \alpha_{3}|\psi|^{2} \partial_{x} \psi+\alpha_{3} \partial_{x}^{3} \psi=0
$$

The soliton solution to this equation is

$$
\psi_{s}(x, t)=a \operatorname{sech}[a(x-c t)] \exp [i(p x-\sigma t)]
$$

with arbitrary $p$ and

$$
c=-3 \alpha_{3} p^{2}+\alpha_{3} a^{2}, \quad \sigma=-\alpha_{3} p^{3}+3 \alpha_{3} p a^{2} .
$$

Then

$$
\Psi_{s}(X, T)=a \operatorname{sech}\left[a\left(X-V_{s} T\right)\right] \exp [i(\kappa X-\omega T)],
$$

where

$$
V_{s}=V+c, \quad \kappa=K+p, \quad \omega=\sigma+\Omega+p V .
$$

Therefore $\kappa$ is arbitrary and $V_{s}(\kappa), \omega(\kappa)$ coincide with Eqs. (2c,d) respectively. This means that Eqs. (74) and (75) indeed describe the Hirota solitons, mentioned in Sec.2a. It is also seen that though the Hirota conditions do not ensure the integrability ( Painlevé tests are negative !), Eq. (1) in Hirota case definitely has exact $N$ -soluton solutions.

Let us now consider numerical solutions of the initial value problem to Eq.(1) in Hirota case

$$
\alpha_{1}=6 \alpha_{3}=-0.6, \quad \alpha_{2}=0 .
$$

As initial condition, we choose a complex pulse

$$
\Psi(X, 0)=A \operatorname{sech}\left[\frac{1}{2}\left(X-X_{0}\right)\right] \exp \left[i C\left(X-X_{0}\right)\right]
$$

with $A=1, C=0.5$ and $X_{0}=85.3$. The structures of $\Psi(X, T)$ at $T=0$ and $T=168$ are shown in Figs. 4(a,b). We see that $\Psi(X, T)$ evolves into two pulses, well separated at $T=168$. The spetrum of $\Psi(X, T)$ at $T=168$ (Fig.5) gives an evidence that, in addition to these pulses, no significant wavetrains are produced at initial condition (77). The time variation of the amplitude of the pulse with largest amplitude, $\left|\Psi^{(1)}\right|$, is shown in Fig.6. It approaches the constant limit, approximately equal to 1.5 . The velocity of that pulse approaches the limit approximately equal to 0.35 . These results suggest that the leading pulse asymptotically approaches a regular soliton with $a^{(1)} \approx 1.5$ and $V_{s}^{(1)} \approx 0.35$. The shapes of $\Psi(X, T)$, obtained numerically for $T>150$, confirm this conclusion. Then, using Eqs. (2c,d) and (4), we can calculate the shifted frequency $\omega^{\prime}=\omega(k)-k V_{s}^{(1)}$, which is the soliton frequency in the reference frame where the soliton is at rest. The plot of $\omega^{\prime}$ versus $V_{s}$, shown in Fig.7, gives $\omega^{\prime}(0.35) \approx 1.5$. This must be equal to $\arg \Psi^{(1)}$. On the other hand, the numerical solution permits to obtain $\arg \Psi^{(1)}$ versus time in the frame where the pulse is at rest. This also gives $\arg \Psi^{(1)} \approx 1.5$, at sufficiently large $T$. Similar results were obtained for the second pulse. Thus we conclude that the initial complex pulse (77) decays into two Hirota solitons. Qualitatively similar results were obtained for the initial pulse

FIG. 4. $\Psi$ versus $x$ at $\alpha_{1}=-0.6, \alpha_{2}=0, \alpha_{3}=-0.1$ and initial condition (77); solid line: $|\Psi|$, dashed line: $R e \Psi$ (a) $T=0$. (b) $T=168$. 
$\Psi(X, 0)=\exp \left[-\frac{\left(X-X_{0}\right)^{2}}{16}+i \frac{X-X_{0}}{2}\right], \quad X_{0}=85.3$.

FIG. 5. $\quad \log _{10}\left|\Psi_{\kappa}\right|$ versus $\kappa$ at $\alpha_{1}=-0.6, \alpha_{2}=0$, $\alpha_{3}=-0.1$ and $T=168$.

FIG. 6. Temporal behavior of the largest soliton amplitude at $\alpha_{1}=-0.6, \alpha_{2}=0, \alpha_{3}=-0.1$ and initial condition (77).

Other features of the dynamics of Hirota solitons, in particular their collisions, will be examined in another paper.

\section{PULSE EVOLUTION AT $\alpha_{1} \neq 6 \alpha_{3}, \alpha_{2}=0$}

Here, we report on the numerical solutions of Eq.(1) in general case. First we take, as initial condition, the pulse (77) with $A=1.9, C=0$ and $X_{0}=100$ and assume that

$$
\alpha_{1}=-0.5, \quad \alpha_{3}=0.1
$$

The behavior of solution at these parameters is shown in Fig.8, where the case $\alpha_{3}=0$ (with $\alpha_{1}=-0.5$ ) is also presented for comparison. In the latter case, the initial pulse splits into four solitons of the form (33), (35)-(38), with $a_{2}=1$ and moving to the left (Fig.8a). On the other hand, in the case (79) the initial pulse splits into three radiating solitons. Two of them propagate to the right and the smallest one - to the left.

FIG. 7. $\omega^{\prime}=\omega-\kappa V_{s}$ versus $V_{s}$ for the largest soliton.
FIG. 8. Development of the initial pulse (77) with $A=1.9$, $C=0, X_{0}=100$ (dashed line). (a) $\alpha_{1}=-0.5, \alpha_{2}=0, \alpha_{3}=0$ $t=48$, (b) $\alpha_{1}=-0.5, \alpha_{2}=0, \alpha_{3}=-0.1 t=48$, (c) $\alpha_{1}=-0.5$, $\alpha_{2}=0, \alpha_{3}=-0.1 t=175$.

FIG. 9. Plots of $X_{0}(t)$ for three largest solitons shown in Fig.8. Dashed line: $\alpha_{1}=-0.5, \alpha_{2}=0, \alpha_{3}=0$; solid line: $\alpha_{1}=-0.5, \alpha_{2}=0, \alpha_{3}=-0.1$

FIG. 10. Fourier spectra of the solutions shown in Fig.8; (a) $\alpha_{1}=-0.5, \alpha_{2}=0, \alpha_{3}=0 ; t=48$ (b) $\alpha_{1}=-0.5, \alpha_{2}=0$, $\alpha_{3}=-0.1 ; t=175$.

The radiating wavetrains propagate to the right with the group velocities larger than the soliton velocities (Figs. 8b and 8c); this is in agreement with Eq. (60). The time dependence of the soliton coordinates is shown in Fig.9 . At $\alpha_{3}$, the soliton velocities are constant. At $\alpha_{3}=-0.1$, the solitons are accelerating in positive direction, which agrees with Eq. (64).

The Fourier spectra for both cases, $\alpha_{3}=0$ and $\alpha_{3}=-0.1$, are shown in Fig. 10. The spectrum at $\alpha_{3}=0$ exhibits no resonant radiation (Fig.10a) while at $\alpha_{3}=-0.1$ we see three distinct resonant peaks with negative $\kappa$, which are the wave numbers of the resonant radiation emitted by the solitons in the "laboratory" frame (Fig.10b). The difference between the wave numbers follows from Eq. (20) which is valid, as we have seen, both for embedded and radiating solitons. As far as the solitons have different velocities and amplitudes (the latters are determined by the soliton parameter $\Lambda$ ), they have different wavenumbers because $\kappa$, being a root of Eq. (20), depends on the soliton velocity and amplitude. 
FIG. 11. The amplitude of the first soliton versus time. (a) $\alpha_{3}=0$, (b) $\alpha_{3}=-0.1$.

FIG. 12. The amplitude of the second soliton versus time. (a) $\alpha_{3}=0$, (b) $\alpha_{3}=-0.1$.

An important difference between the two cases, $\alpha_{3}=0$ and $\alpha_{3} \neq 0$, is seen in Figs. 11 and 12. In the first case, the average soliton amplitudes have constant limits at $t \rightarrow \infty$ while in the second case they must slowly decrease because of the soliton radiation.

In the aforegoing, we studied the case when $\alpha_{1} \alpha_{3} \geq 0$. Now let us consider $\alpha_{1} \alpha_{3} \leq 0$. We take

$$
\alpha_{1}=-0.6, \quad \alpha_{3}=0.1
$$

FIG. 13. The RRS at parameters $\alpha_{1}=-0.6, \alpha_{3}=0.1$. Full line: $|\Psi|$, dotted line : $\operatorname{Re} \Psi$.

\section{FIG. 14. Spectral distribution at $\alpha_{1}=-0.6, \alpha_{3}=0.1$.}

and initial condition (77) with $C=1 / 2, X_{0}=850$. The solution at $T=370$ is shown in Fig. 13 . We see a pulse with radiation on both its sides. An analysis shows that on the left hand side there is the resonantly generated radiation (in agreement with Eq. (60), where now $\left.\alpha_{3}>0, k>0, U<0\right)$. On the right hand side, there is a radiation composed from the harmonics of continuous spectrum; it satisfies the linearized Eq.(1) and has been emitted in the transient period of time. The spectal distribution is shown in Fig.14. One can see two spectral maximums at positive wavenumbers. The narrow one corresponds to the resonant radiation, while the broader peak is composed of the continuous spectrum. Its structure can be understood from the dispersion equation

$$
\omega=(1 / 2) k^{2}-\alpha_{3} k^{3}
$$

and the correspondig expression for the group velocity of continuous spectrum

$$
V_{g}(k)=k-3 \alpha_{3} k^{2} .
$$

From this we see that, at $\alpha_{3}>0$, the continuos radiation on the right hand side of the pulse is composed from

$$
0<k \leq k_{0}=1 / 6 \alpha_{3}
$$

where $V_{g}\left(k_{0}\right)=\max V_{g}(k)$. As far as $k_{0}$ is less then the wavenumber of resonant radiation, approximately given by Eq.(50) (note that in our case , according to (45), $a_{2}>1$ ), one can see why the peak of continuous spectra is less than $\kappa$ of resonant radiation. All this shows that the pulse in Fig. 13 is nothing than RRS. The time behavior of its amplitude is shown in Fig. 15 ; qualitatively, it is similar to Fig. 3a.

It is reasonable to compare these results with the case $\alpha_{1}=-0.6, \alpha_{3}=0$, at the same initial condition. Then we have two regular solitons, propagating to the right. The soliton positions versus time for both cases are shown in Fig. 16. Note a very small acceleration of RRS in the direction of group velocity (which is now negative) .

FIG. 15. Soliton amplitude versus time at $\alpha_{1}=-0.6, \alpha_{3}=0.1$.

\section{CONCLUSIONS}

We have considered soliton and quasisoliton solutions of Eq. (1) and their relationship. Solutions (2), describing regular solitons, degenerate at $\alpha_{3} \rightarrow 0$; this shows that they exist due a balance between nonlinear terms and linear third order dispersion. On the other hand the quasisolitons (embedded and resonantly radiating) 
This work was partly supported from INTAS grant No 99-1068.

\section{Appendix A}

\section{Modulational instability of plane wave, accord- ing to Eq.(1)}

FIG. $\quad 16$ ton positions versus time for $\alpha_{1}=-0.6, \alpha_{3}=0(1,2)$ and, $\alpha_{1}=-0.6, \alpha_{3}=0.1(3)$.

turn at $\alpha_{3} \rightarrow 0$ into the regular soliton solutions of Eq. (1) without the third derivative term. Apart from that, at $\alpha_{2}=0$, the regular solitons exist only in the special case $\alpha_{1}=6 \alpha_{3}$.

The resonantly radiating solitons (RRS) are nonsteady; the amplitudes of their radiation at small $\alpha_{3}$ are exponentially small and so their parameters change logarithmically slow in this case. Only such quasisteady solitons have sufficiently large lifetimes to be of physical significance. The embedded solitons (ES), which are steady structures consisting of pulses embedded into plane waves (wings), have close connection with RRS, similar to other systems. The corresponding analytical treatment in Sec.III is suplemented by numerical simulations with the cut off operation (Sec.IV) ; it is demonstrated that it leads to the transformation of ES into RRS that radiates in the direction of the radiation group velocity . Just after the cutt off, the amplitude, velocity and wavenumber of the radiated wavetrain coincide with those of wings; marching in time, we have seen that the amplitude decreases, the soliton velocity increases and the wavenumber changes according to Eqs.(30) and (31). This is in agreement with conservation laws (Appendix B).

In Sec. $\mathrm{V}$ we have investigated a special case $\alpha_{1}=6 \alpha_{3}$, interesting from a theoretical point of view. Then the quasisolitons, ES and RRS, do not exist and Eq. (1) can be reduced to the complex MKdV equation which has $N$-soliton solutions (consisting of regular solitons). This generalizes the Hirota solutions obtained for $\alpha_{1}=$ $6 \alpha_{3}, \alpha_{2}=0$. The complex MKdV equation possesses the Painlevé property only at $\alpha_{2}=3 \alpha_{3}$, which is the case when Eq.(1) is integrable.

Presumably, in the nonlinear processes only regular solitons and RRS may be of the physical significance. From Eq. (2b) it follows, however, that the amplitude of regular soliton vanishes at $\alpha_{3} \rightarrow 0$, while for the RRS it remains finite. Therefore one should expect that at small $\alpha_{3}$, the RRS are more important in the nonlinear evolution. This was confirmed by solving the initial value problem (Sec. VI), where the RRS and not regular solitons are seen emerging from the initial disturbances. (Small $\alpha_{3}$ is the most interesting case from physical point of view because the third derivative term is in fact the result of an expansion).
Eq. (1) has exact plain wave solution

$$
\Psi=A \exp (i \kappa X-i \omega T) .
$$

Substituting (A1) into (1), we have

$$
\omega=\frac{1}{2} \kappa^{2}-\left(1-\alpha_{1} \kappa\right) A^{2}-\alpha_{3} \kappa^{3} .
$$

Substituting a slightly perturbed wave (A1)

$$
\Psi=A(1-\chi) \exp (i \kappa X-i \omega T)
$$

into Eq.(1), we have

$$
\begin{aligned}
& i \chi_{T}+\frac{1}{2}\left(1-6 \alpha_{3} K\right) \chi_{X X}+i\left(K+\alpha_{1} A^{2}-3 \alpha_{3} K^{2}\right) \chi_{X} \\
& \quad+\left(1-\alpha_{1} \kappa\right)\left(\chi+\chi^{*}\right) A^{2}+i \alpha_{2}\left(\chi_{X}+\chi_{X}^{*}\right) A^{2} .
\end{aligned}
$$

Writing $\chi=u+i w$ and assuming that

$$
(u, w) \approx \exp (i p X-i r T) .
$$

we obtain dispersion equation, which is convenient to write in the form

$$
\begin{gathered}
\Gamma^{2}-2 \alpha_{2} k A^{2} \Gamma-\frac{1}{4}\left(1-6 \alpha_{3} K\right) k^{2}\left[\left(1-6 \alpha_{3} K\right) k^{2}\right. \\
\left.-4\left(1-\alpha_{1} K\right) A^{2}\right]=0
\end{gathered}
$$

where

$$
\Gamma=r+\alpha_{3} p^{3}-\left(\kappa-3 \alpha_{3} \kappa^{2}+\alpha_{1} A^{2}\right) p .
$$

Thus, at real $p, \operatorname{Im} \gamma=\operatorname{Im} r$ and the stability condition is

$$
\left(1-6 \alpha_{3} \kappa\right)^{2} p^{2} \geq 4 A^{2}\left[\left(1-\alpha_{1} \kappa\right)\left(1-6 \alpha_{3} \kappa\right)-\alpha_{2}^{2} A^{2}\right] .
$$

Unlike the plain wave solution of the regular NLS, now the plain wave can be stable at any $p$, if

$$
\left(1-\alpha_{1} \kappa\right)\left(1-6 \alpha_{3} \kappa\right) \leq \alpha_{2}^{2} A^{2} .
$$

At $\alpha_{2}=0$ this is possible at

$$
\begin{aligned}
& \frac{1}{\alpha_{1}} \leq \kappa \leq \frac{1}{6 \alpha_{3}} \quad\left(6 \alpha_{3}<\alpha_{1}\right) \\
& \frac{1}{6 \alpha_{3}} \leq \kappa \leq \frac{1}{\alpha_{1}} \quad\left(\alpha_{1}<6 \alpha_{3}\right)
\end{aligned}
$$


Applying the stability criterion to the wings of embedded solitons, where $\kappa \approx 1 / 2 \alpha_{3}$, we see that this may satisfy condition $(\mathrm{A} 9 \mathrm{~b})$; then one can expect that the wings are stable. We should also take into account only $p>L^{-1}$, where $L$ is the period in the numerical scheme; this relaxes the limitatations following from the stability criterion.

\section{Appendix B}

\section{Investigation of the soliton evolution by means} of the integrals of motion.

First, we present a general analysis of conserving integrals $N, P, H$. Substituting (5) into expressions (12), (14), (15) we have after simple algebra

$$
\begin{gathered}
N=\int_{\infty}^{\infty}|\psi(x, t)|^{2} d x, \\
P=K N+\frac{1}{2} i \int_{\infty}^{\infty}\left(\psi \partial_{x} \psi^{*}-\psi^{*} \partial_{x} \psi\right) d x .
\end{gathered}
$$

where $\psi(x, t)$ satisfies Eq.(9).

To have a convenient expression for $H$, we substitute in Eq. (15) $\alpha_{3} \partial_{x}^{3} \Psi$ and $\alpha_{3} \partial_{x}^{3} \Psi^{*}$ from Eq.(1), to obtain

$$
\begin{aligned}
H= & \frac{i}{2} \int_{\infty}^{\infty} d X\left(\Psi^{*} \partial_{T} \Psi-\Psi \partial_{T} \Psi^{*}\right)+\frac{1}{2} \int_{\infty}^{\infty} d X|\Psi|^{4} \\
& +\frac{i}{4} \alpha_{1} \int_{\infty}^{\infty} d X|\Psi|^{2}\left(\Psi^{*} \partial_{X} \Psi-\Psi \partial_{X} \Psi^{*}\right) .
\end{aligned}
$$

Then using the Galilei transformation in the form (24) and taking into account that at large $t$

$$
\partial_{t} \approx i\left(\lambda^{2} / 2\right) \psi,
$$

we arrive at the following asymptotic expression

$$
\begin{aligned}
H \approx( & \left(\Omega-K V-\frac{1}{2} \lambda^{2}\right) N+P V+\frac{1}{2} q \int_{\infty}^{\infty}|\psi|^{4} d x \\
& -\frac{1}{2} i \alpha_{1} \int_{\infty}^{\infty}|\psi|^{2}\left(\psi \partial_{x} \psi^{*}-\psi^{*} \partial_{x} \psi\right) d x
\end{aligned}
$$

Substituting (25) and (32) into (B1), we have at large $t$ (cf. Refs. [25,26]),

$$
N \approx N_{s}+N_{r}
$$

where

$$
N_{s}=\int_{\infty}^{\infty}\left|u_{s}(x, t)\right|^{2} d x, \quad N_{r}=\int_{\infty}^{\infty}|\eta(x, t)|^{2} d x
$$

are contributions from the soliton and the radiation. Using (35) we have

$$
N_{s}=\frac{2 a_{2}}{\alpha_{1}} \arctan \left(\frac{\alpha_{1} \lambda}{\sqrt{a_{2}} q}\right)
$$

and, at large $t$,

$$
N_{r} \approx|f|^{2}|U|_{t}
$$

where $f$ is given by (52) or (55). Evidently, $|f|^{2}$ does not depend on $x$. Then from the conservation of $N$ it follows

$$
\frac{d}{d t}\left[\frac{2 a_{2}}{\alpha_{1}} \arctan \left(\frac{\alpha_{1} \lambda}{\sqrt{a_{2}} q}\right)\right] \approx-|f|^{2}|U| .
$$

In a similar way, from (B2) and the conservation of $P$ we have the following asymptotic equation at large $t$

$$
N \frac{d K}{d t}+\frac{d}{d t} \int_{\infty}^{\infty} u_{s}^{2}(x) \partial_{x} \psi_{s}(x) d x+k|f|^{2}|U| \approx 0 .
$$

From (36) at $\alpha_{2}=0$, we have

$$
\partial_{x} \psi_{s}(x)=-\frac{\alpha_{1}}{2 a_{2}} u_{s}^{2}(x) .
$$

Taking into account that

$$
\int_{\infty}^{\infty} u_{s}^{4}(x) d x=\frac{2 a_{2} q}{\alpha_{1}}\left(\frac{2 \sqrt{a_{2}} \lambda}{q}-N_{s}\right),
$$

we transform Eq. (B9) to the form

$$
N \frac{d K}{d t}-\frac{d}{d t}\left[\frac{q}{\alpha_{1}}\left(\frac{2 \sqrt{a_{2}} \lambda}{q}-N_{s}\right)\right]+k|f|^{2}|U| \approx 0 .
$$

Now we turn to Eq.(B3). After differentiation over $t$ and simple calculations we obtain

$$
P \frac{d V}{d t}-\left(\lambda \frac{d \lambda}{d t}+a_{2} K \frac{d K}{d t}\right) N
$$

$$
\begin{gathered}
=-\frac{d}{d t}\left[\frac{1}{2} q \int_{\infty}^{\infty}|\psi|^{4} d x+\frac{i}{4} \alpha_{1} \int_{\infty}^{\infty}|\psi|^{2}\left(\psi^{*} \partial_{x} \psi-\psi \partial_{x} \psi^{*}\right) d x\right] \\
=-\frac{d}{d t}\left[\frac{1}{2} q \int_{\infty}^{\infty}\left|\psi_{s}\right|^{4} d x+\frac{i}{4} \alpha_{1}\right. \\
\left.\int_{\infty}^{\infty}\left|\psi_{s}\right|^{2}\left(\psi_{s}^{*} \partial_{x} \psi_{s}-\psi_{s} \partial_{x} \psi_{s}^{*}\right) d x\right] .
\end{gathered}
$$

Using

$$
\frac{d V}{d t}=a_{2} \frac{d K}{d t}
$$

we obtain

$$
a_{2}(P-K N) \frac{d K}{d t}-\lambda \frac{d \lambda}{d t} N \approx
$$




$$
-\frac{d}{d t}\left(\frac{1}{2} q \int_{\infty}^{\infty} u_{s}^{4} d x+\frac{\alpha_{1}^{2}}{2 a_{2}} \int_{\infty}^{\infty} u_{s}^{6} d x\right) .
$$

Let us apply these relations to small $\alpha_{1}$ (which is just the case shown in Figs.3). Assuming that

$$
\frac{\alpha_{1} \lambda}{\sqrt{a_{2}} q} \ll 1
$$

we have

$$
N_{s} \approx \frac{2 \sqrt{a_{2}} \lambda}{q}\left(1-\frac{\alpha_{1}^{2} \lambda^{2}}{3 a_{2} q^{2}}\right) .
$$

Therefore Eq. (B8) is reduced to

$$
\frac{d}{d t}\left[\frac{2 \sqrt{a_{2}} \lambda}{q}\left(1-\frac{\alpha_{1}^{2} \lambda^{2}}{3 a_{2} q^{2}}\right)\right]=-|f|^{2}|U| .
$$

In the same approximation, Eq.(12) takes the form

$$
N \frac{d K}{d t} \approx-k|f|^{2}|U|+\frac{2 \alpha_{1}}{3} \frac{d}{d t}\left(\frac{\lambda^{3}}{\sqrt{a_{2}} q^{2}}\right),
$$

At $\alpha_{1} \rightarrow 0$, Eqs. (B18) and (B19) turn into equations obtained in Ref.[18]. From (B19), (B14) and Eq. (60) it follows an important qualitative result (64).

Now, consider Eq. (B15). Taking into account (B2),(B10), (B11) and (B19) we see that at

$$
t \ll \lambda k^{-2}|f|^{-4} U^{-2}
$$

and small $\alpha_{1}$, Eq. (B15) is approximately equivalent to (B18). Performing calculations similar to those in Ref. [19] and using (B14), we conclude that the soliton width $\lambda^{-1}$ and velocity $V(t)$ are changing logarithmically slow. The same holds for the soliton amplitude $u(9 t)$, determined from (39) and (37), in agreement with numerical solution of Eq.(1) (see Figs.3). The chracteristic time of the variations of soliton parameters has the same order of magnitude as in Ref.[26], which is much less than the right hand side of (B20).

If $\alpha_{1}$ is not small, the analysis is rather tedious and will not be considered here.

\section{References}

[1] A. Hasegawa and Y. Kodama, Solitons in Optical Communications (Oxford University Press, 1995).

[2] G.P. Agrawal, Nonlinear Fiber Optics (Academic Press, New York, 1995).

[3] M.J. Potasek and M.Tabor, Phys.Lett. A 154, 449 (1991).

[4] R.Hirota, J. Math.Phys. 14,805 (1973).

[5] N.Sasa and J.Satsuma, J. Phys.Soc. Jpn. 60 ,409 (1991).

[6] E.M. Gromov, L.V. Piskunova and V.V.Tutin , Phys. Lett. A 256, 153 (1999) .

[7] J.Yang, B.A.Malomed and D.J. Kaup, Phys. Rev. Lett. 83, 1958 (1999).
[8] P.K.A.Wai, C.R.Menyuk, Y.C.Lee and Chen, Opt.Lett.11, 464 (1986).

[9] P.K.A.Wai, H.H.Chen and Y.C.Lee, Phys.Rev. A 41, 426 (1990).

[10] H.H. Kuehl and C.Y. Zhang, Phys.Fluids B 2, 889 (1990).

[11] V.I. Karpman, Phys. Rev.E , 47, 2073 (1993) .

[12] J.P. Boyd,Weakly Nonlocal Solitary Waves and Beyond-All_Order Asymptotics (Kluver, Dodrecht,1998).

[13] V.I. Karpman, In : Nonlinearity, Integrability and all that:Twenty years after NEEDS'r9. Editors M.Boiti, L.Martina, F.Pempinelli, B. Prinari \& G. Soliani, ("World Scientific", 2000), p.396.

[14] V.I. Karpman, Phys.Rev. E 62 , 5678 (2000).

[15] P.Deeskow, H.Schamel, N.N.Rao, M.Y. Yu, R.K. Varma and P.K.Shukla, Phys. Fluids 30 , 2703 (1987).

[16] V.I. Karpman and H. Schamel, Phys. Plasmas 4,120 (1997); ibid.4 (1997) 2778 (E) .

[17] Y. Pomeau, A.Ramani and B. Grammaticos, Physica D 31 (1988) 127.

[18] V.I.Karpman, Phys. Lett.A 181 (1993) 211.

[19] V.I. Karpman, Phys.Lett.A 186 (1994) 300.

[20] V.I.Karpman, Phys.Rev.Lett. 74 (1995) 2455.

[21] J. Weiss, M.Tabnd G. Carnevale, J. Math. Phys. 24, 522 (1983) .

[22] M.A. Ablowitz and H. Segur Solitons and the Inverse Scattering Transform (SIAM, Philadelphia, 1981)

[23] M. Gedalin, T.C. Scott and Y.B. Band, Phys. Rev. Lett. 78, 448 (1997).

[24] V. E. Zakharov and E.A. Kuznetsov, JETP 86,1036 (1998).

[25] V.I. Karpman and A.G. Shagalov, Phys. Lett. A 254, 319 (1999).

[26] V.I.Karpman, Phys. Lett. A 244 , 397 (1998) .

[27] Zhonghao Li, Lu Li, Huipping Tian and Guosheng Zhou, Phys.Rev.

Lett. 84,4096 (2000).

[28] K. Portezian and K. Nakkeeran, Phys.Rev.Lett. 76, 3955 (1996) 
This figure "FIG1.jpg" is available in "jpg" format from: http://arxiv.org/ps/nlin/0102008v1 
This figure "Fig2a.jpg" is available in "jpg" format from: http://arxiv.org/ps/nlin/0102008v1 
This figure "fig2b.jpg" is available in "jpg" format from: http://arxiv.org/ps/nlin/0102008v1 
This figure "fig2c.jpg" is available in "jpg" format from: http://arxiv.org/ps/nlin/0102008v1 
This figure "fig3a.jpg" is available in "jpg" format from: http://arxiv.org/ps/nlin/0102008v1 
This figure "fig3b.jpg" is available in "jpg" format from: http://arxiv.org/ps/nlin/0102008v1 
This figure "Fig4a.jpg" is available in "jpg" format from: http://arxiv.org/ps/nlin/0102008v1 
This figure "Fig4b.jpg" is available in "jpg" format from: http://arxiv.org/ps/nlin/0102008v1 
This figure "Fig5.jpg" is available in "jpg" format from: http://arxiv.org/ps/nlin/0102008v1 
This figure "Fig6.jpg" is available in "jpg" format from: http://arxiv.org/ps/nlin/0102008v1 
This figure "Fig7.jpg" is available in "jpg" format from: http://arxiv.org/ps/nlin/0102008v1 
This figure "Fig8.jpg" is available in "jpg" format from: http://arxiv.org/ps/nlin/0102008v1 
This figure "Fig9.jpg" is available in "jpg" format from: http://arxiv.org/ps/nlin/0102008v1 
This figure "Fig10.jpg" is available in "jpg" format from: http://arxiv.org/ps/nlin/0102008v1 
This figure "Fig11.jpg" is available in "jpg" format from: http://arxiv.org/ps/nlin/0102008v1 
This figure "Fig12.jpg" is available in "jpg" format from: http://arxiv.org/ps/nlin/0102008v1 
This figure "Fig13.jpg" is available in "jpg" format from: http://arxiv.org/ps/nlin/0102008v1 
This figure "Fig14.jpg" is available in "jpg" format from: http://arxiv.org/ps/nlin/0102008v1 
This figure "Fig15.jpg" is available in "jpg" format from: http://arxiv.org/ps/nlin/0102008v1 
This figure "Fig16.jpg" is available in "jpg" format from: http://arxiv.org/ps/nlin/0102008v1 